\title{
Configuration of ONE Simulator using Eclipse
}

\author{
Atul Sharma ${ }^{1}$, Piyush Gupta ${ }^{2}$, Jitender Grover ${ }^{3}$ \\ ${ }^{1,2}$ (Department of CSE, UIET, Kurukshetra University Kurukshetra) \\ ${ }^{3}$ (Department of Computer Science \& Engineering, MM University, Sadopur, Ambala, Haryana, India) \\ ('atulsharma2204@gmail.com, ${ }^{1}$ piyushgpt.er@gmail.com, ${ }^{3}$ jitendergrover0101@gmail.com)
}

\begin{abstract}
One is a simulation tool specially designed for DTN.ONE provides simulation environment for complete wireless networks like VANET, MANET etc. ONE provides different routing schemes and movement models. You can easily use them and perform simulation of theme according to your requirements, Using ONE simulator you can easily create new routing scheme and new movement model. Also you can easily implement and simulate Different types of security attacks and their removal mechanisms. Eclipse is also java based tool used for programming purpose. In this paper how to configure ONE simulator with eclipse is described with proper steps. Configuring one simulator with eclipse helps in programming of different routing scheme and movement models because eclipse provides some built-in functions of java that helps in programming.
\end{abstract}

Keywords: ONE (Opportunistic Network Environment), DTN (Delay Tolerance Network), VANET (Vehicular Ad hoc Network), MANET (Mobile Ad hoc Network), Eclipse, JDK.

\section{INTRODUCTION}

One is a Java based simulator targeted for research in Delay Tolerant Networks (DTNs). The ONE simulator has been developed in the SINDTN and CATDTN projects supported by Nokia Research Center (Finland). ONE provides facility to users to generate information from the simulation that they performed. This simulation environment written in Java is completely configurable and is able to completely simulate the behavior of nodes in the simulations that includes movement of nodes, connections between nodes and provides complete routing information of each node. A more interesting feature, for our purposes, is the ability of emulating message routing using different routing protocols.

To emulate nodes movement, the ONE can take in input traces from real time dimensions. One characteristic of the ONE architecture is high modularity, as shown in Figure 1. Each movement models and for each routing protocol is implemented in an independent component, that are loaded at run time depending on the simulator parameters settings. In ONE simulator it is easy to implement new movement model and new routing protocols in the simulator.

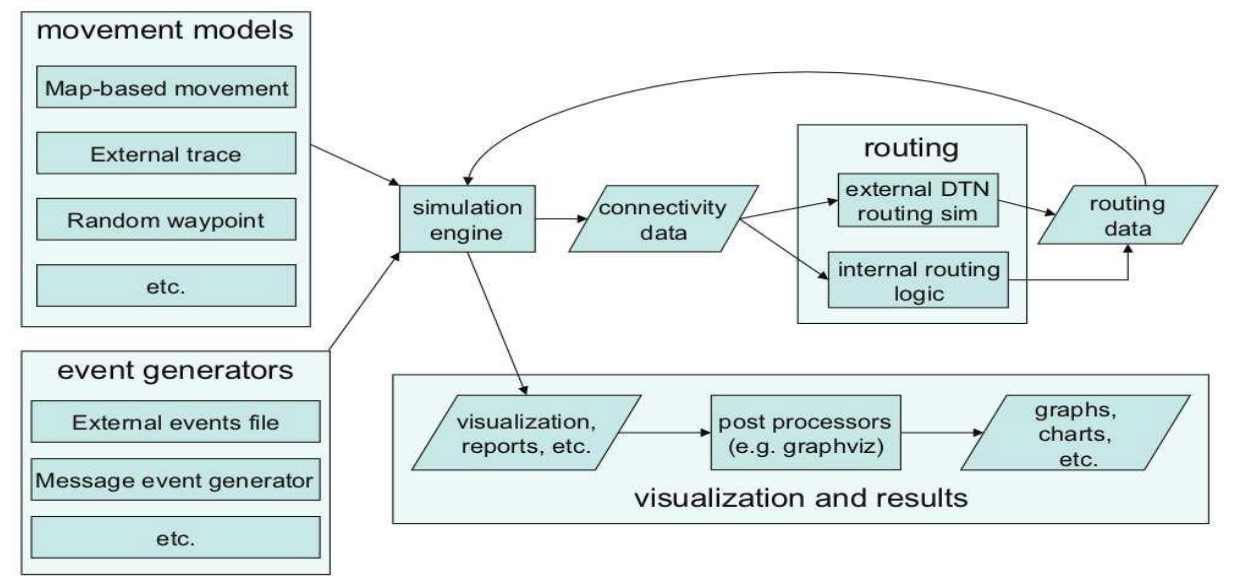

Fig.1: Overview of the ONE simulation environment [2] 


\section{SIMULATION}

a) Algorithm development

b) Modeling, simulation, and prototyping

c) Data analysis, exploration, and visualization

d) Scientific and engineering graphics

e) Application development, including Graphical User Interface building

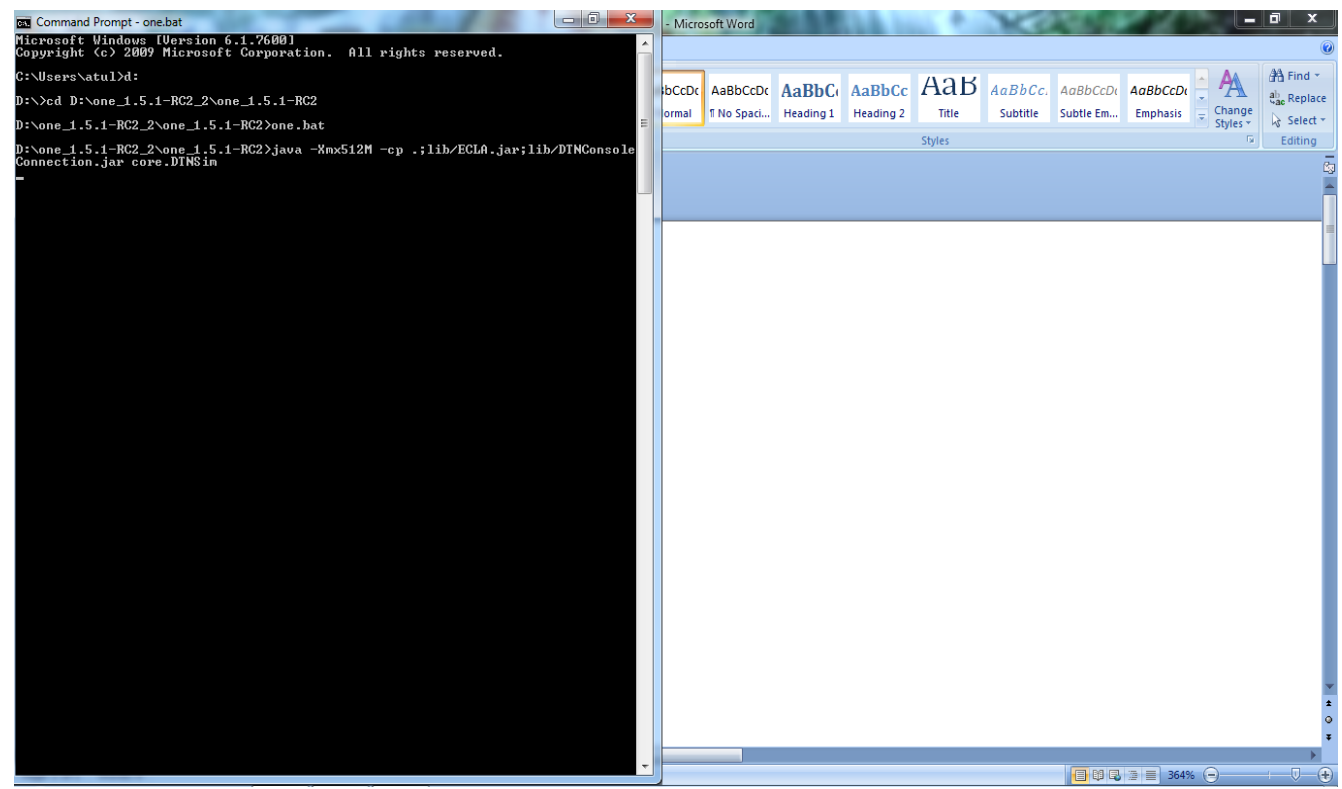

Fig. 2: command mode of ONE Simulator

In Figure 2, shown how one simulator is run using command prompt? Here first set the path of one simulator and then ONE.BAT file. Now simulator will run.

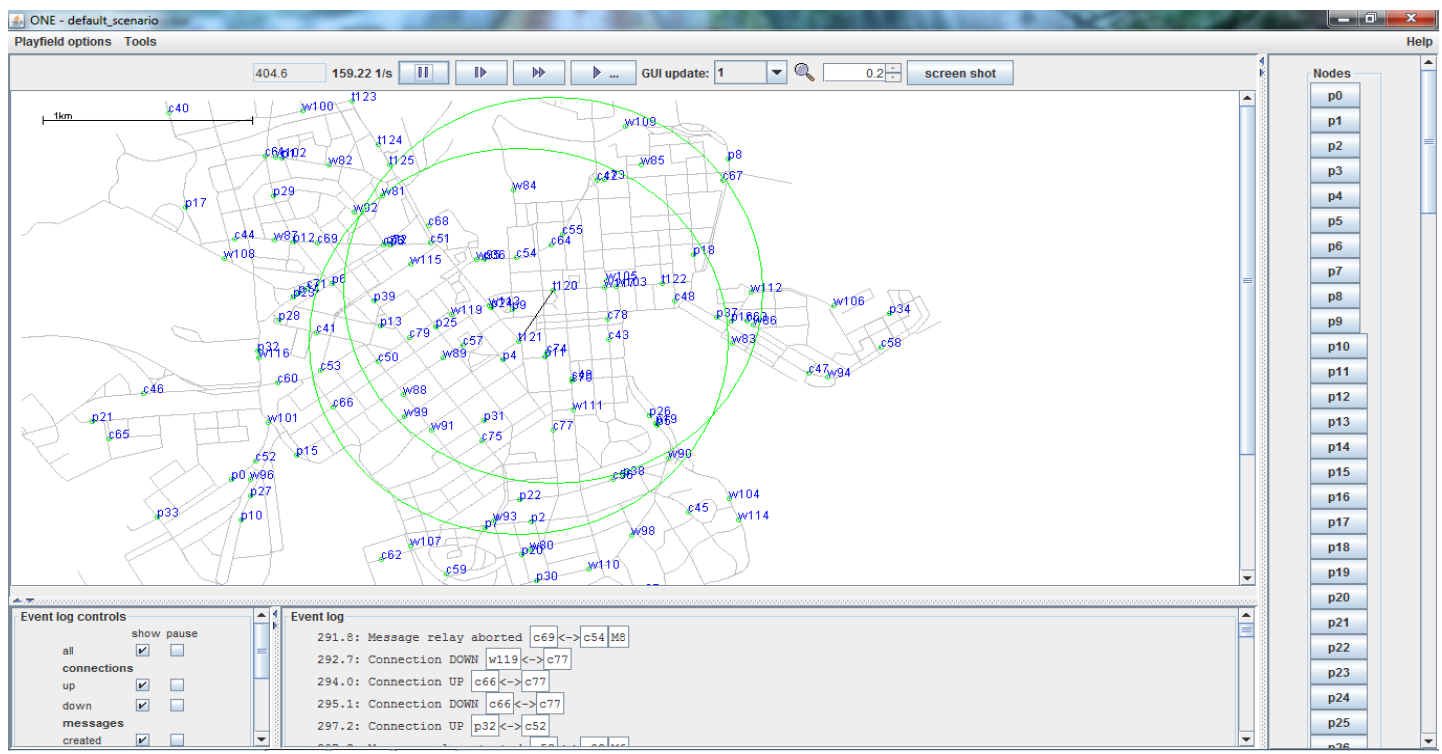

Fig. 3: GUI mode of ONE Simulator 
Figure 3 shows the GUI window of ONE Simulator. By clicking on run button simulation will start. Number of nodes movement is shown in figure.

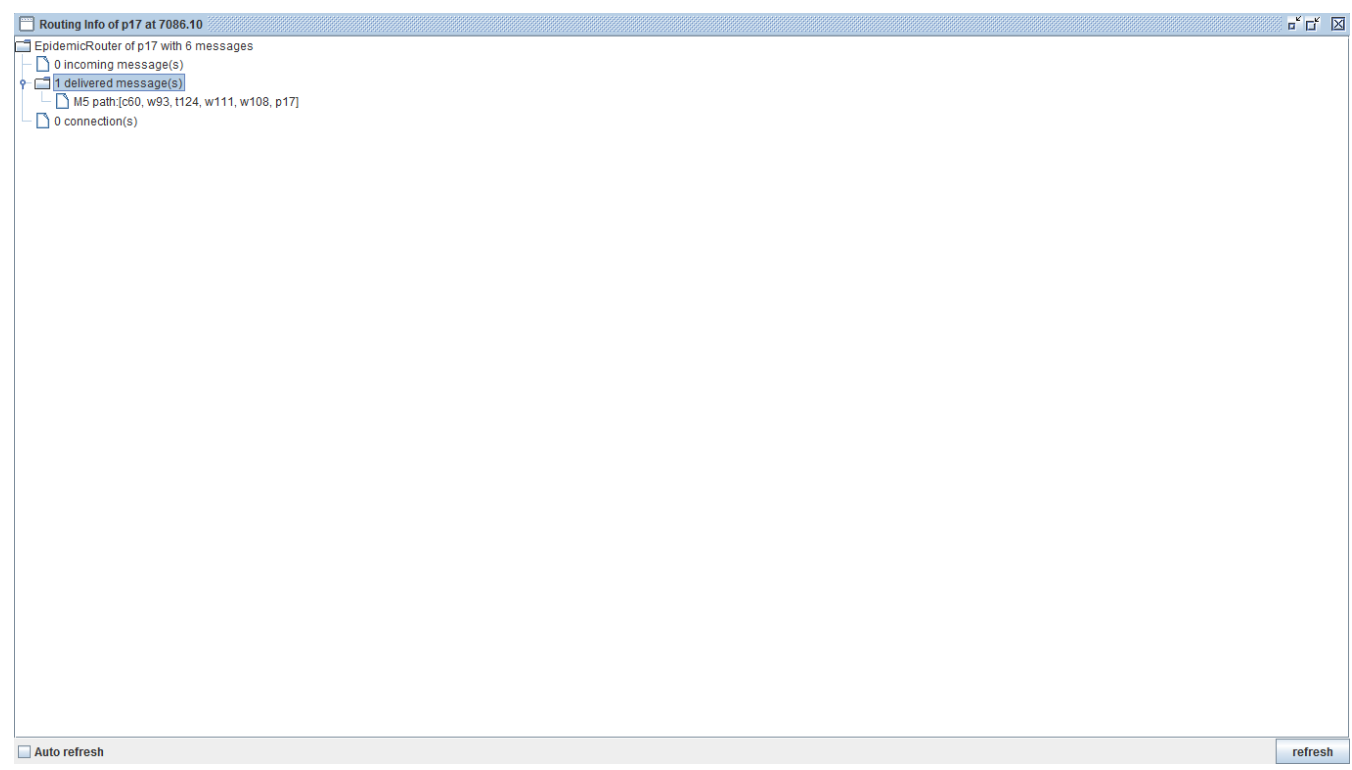

Fig. 4: Routing information of node

Figure 4 shows routing information of node. Routing information consider number of packets contained by node, number of packets delivered by node, number of connection.

\section{CONFIGURATION WITH ECLIPSE}

This section explains how to configure ONE Simulator with eclipse. Configuring ONE Simulator with eclipse helps in programming. Eclipse helps in programming by providing some built in functions. Also you can run multiple projects in ONE simulator using eclipse. For configuring you first installed JDK1.6 then install eclipse. After doing this follow following steps to configure ONE Simulator.

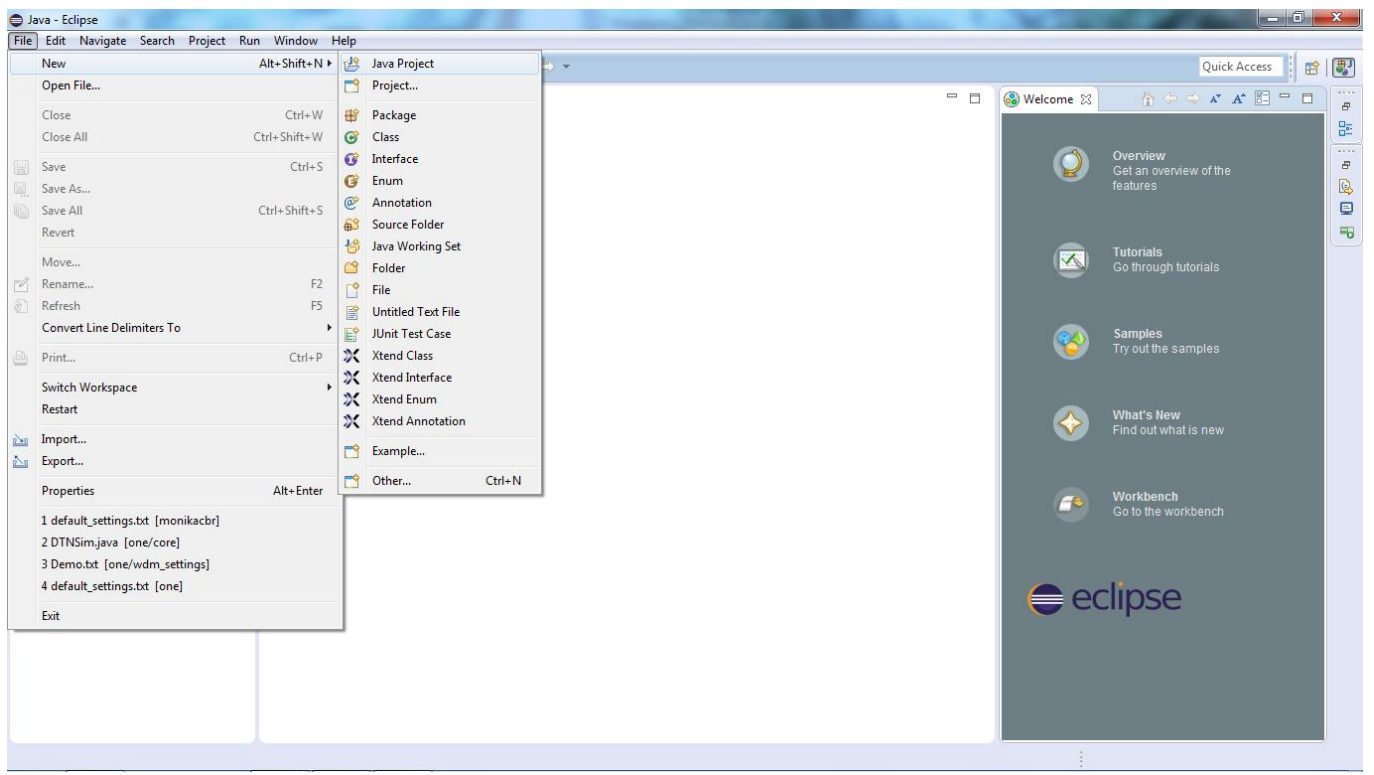

Fig. 5: Eclipse GUI 
Open eclipse then go to file> java project by New>Java project.

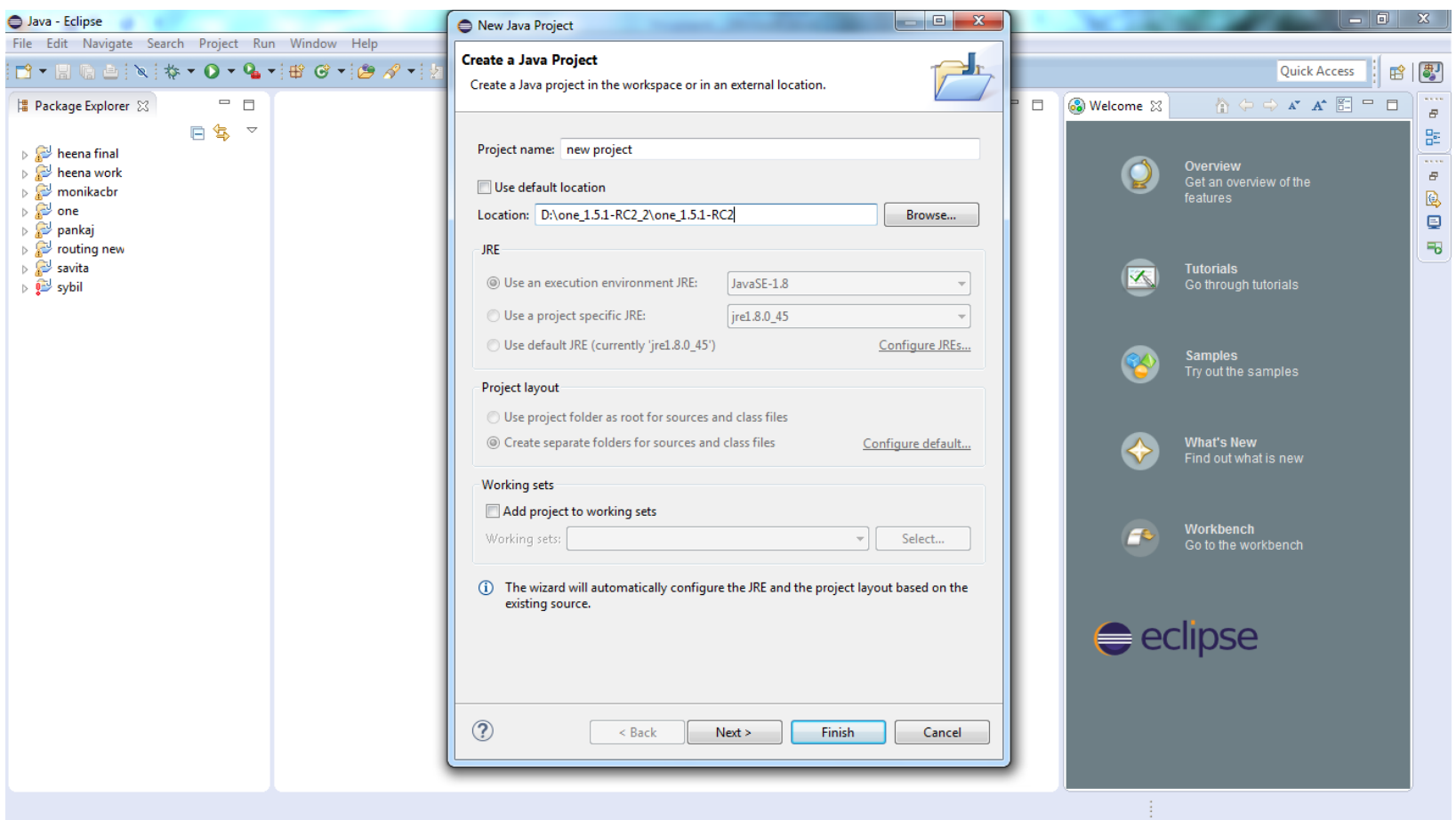

Fig. 6: depicting the path of node

A popup window is open in this enter the name of project like new project then set location of ONE Simulator

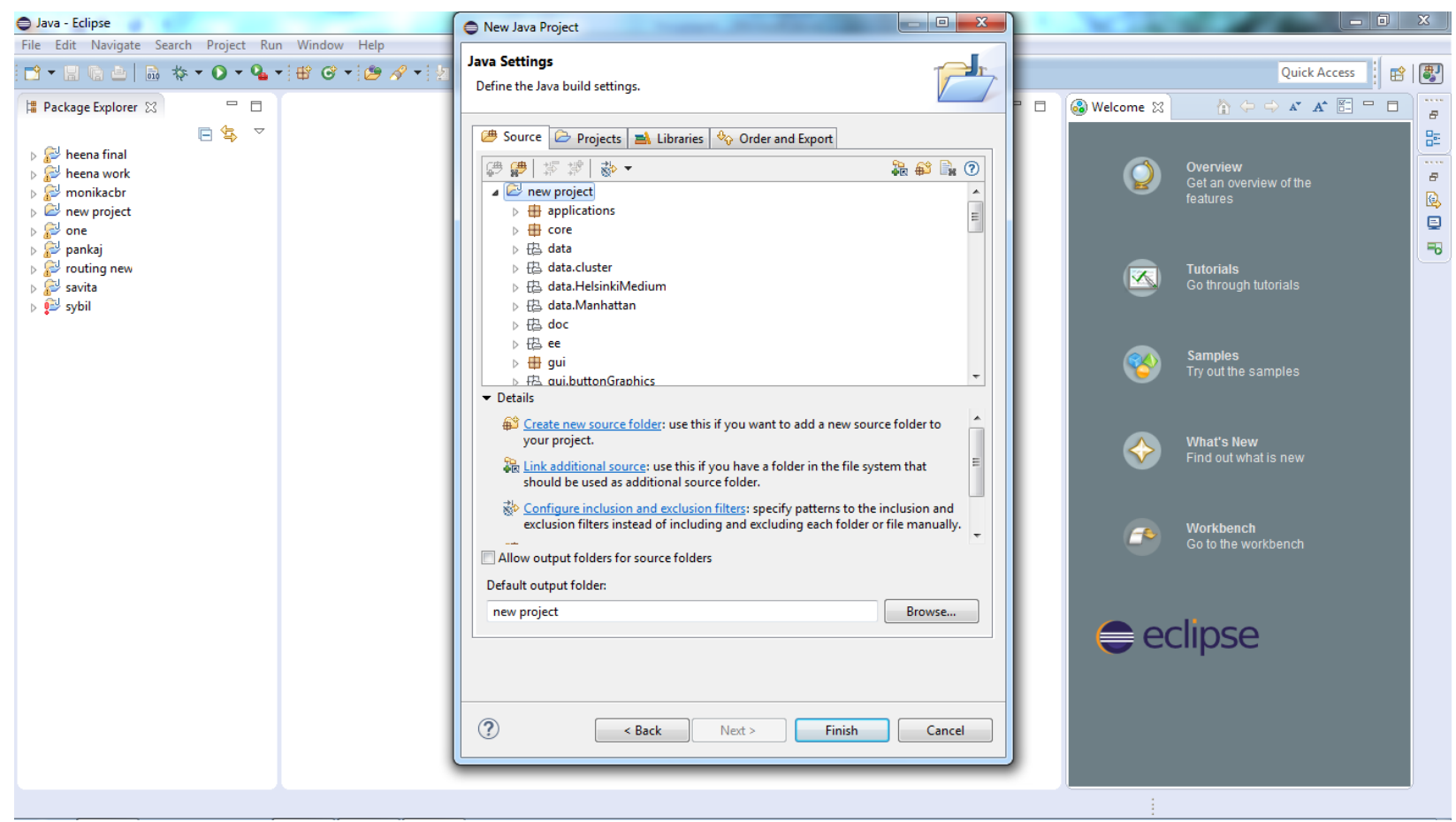

Fig. 7: Depicting new java project includes ONE simulator packages 
Then click on next button again open a popup which show the folders of ONE Simulator. Now clock on finish button this will create your new project in eclipse.

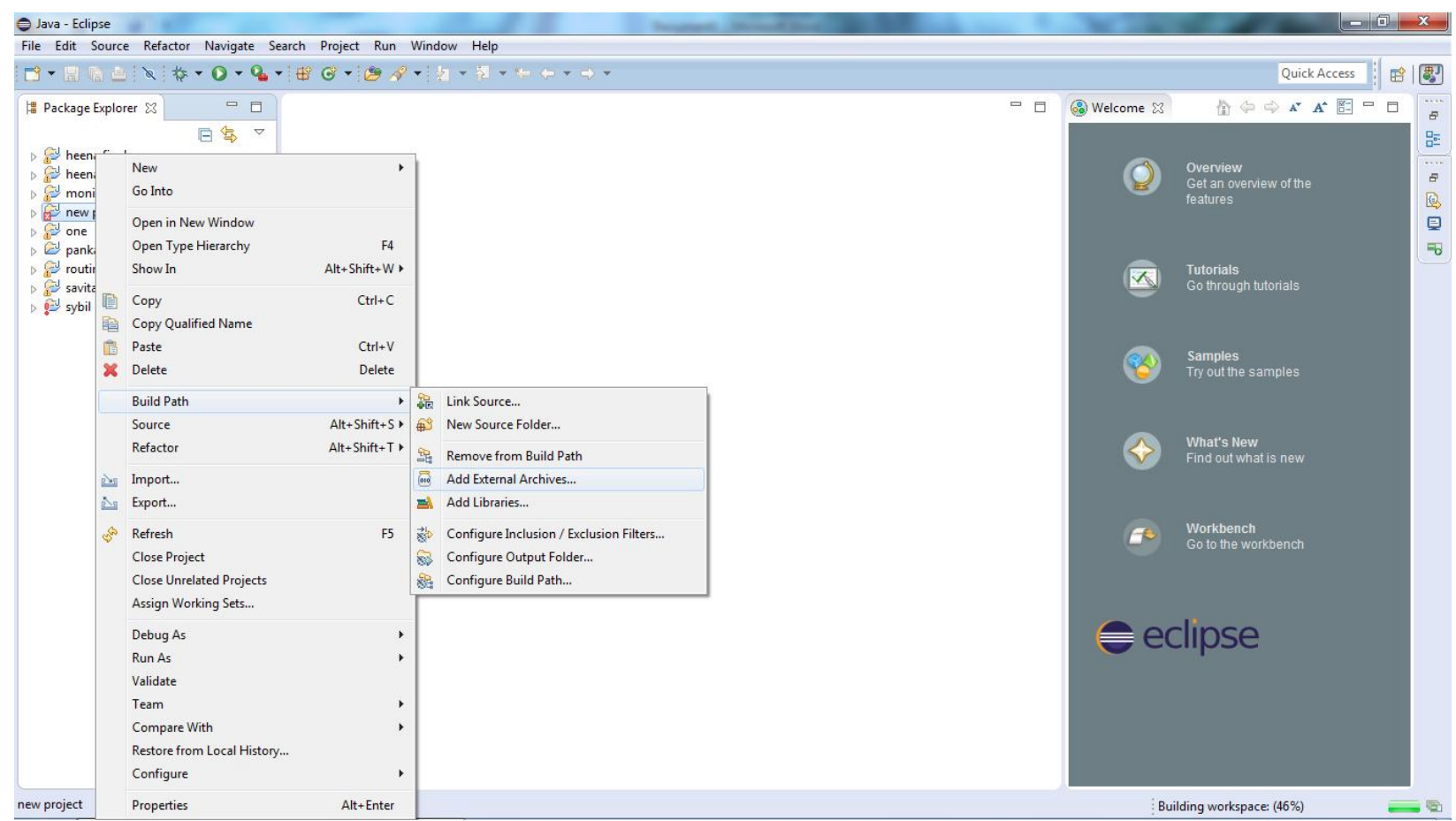

Fig. 8: depicting how build path is set.

Now right click on new project and go to build path>add external archives to set library files. There are three library files named DTNConsoleConnection.jar, Ecla.jar and Junit-4.11.jar.

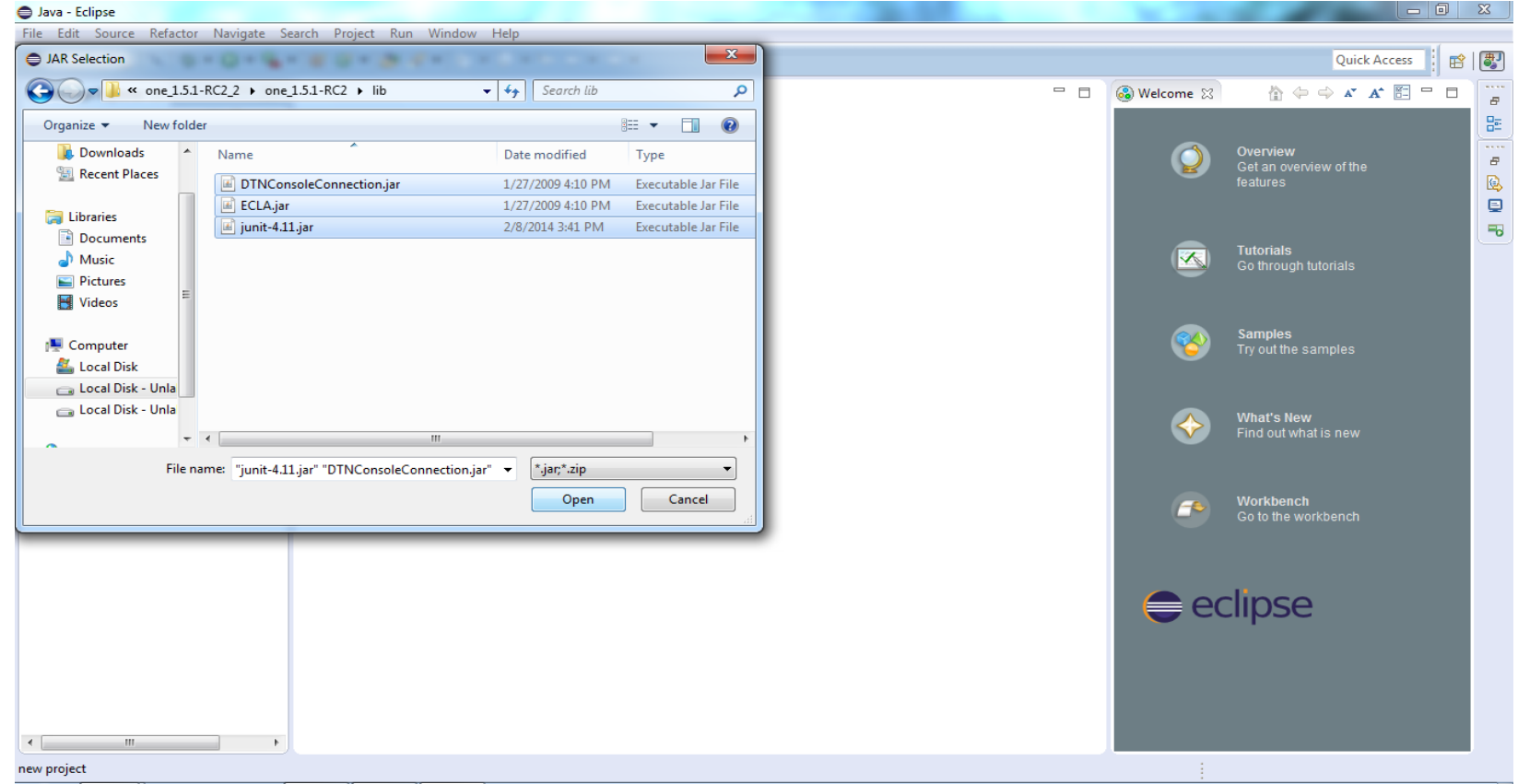

Fig. 10: depicting how library files are included 
Now a popup window is open in which you set path of above mentioned three library files. These files rely in lib folder of ONE Simulator. Now click on OK button your simulator is configured with eclipse.

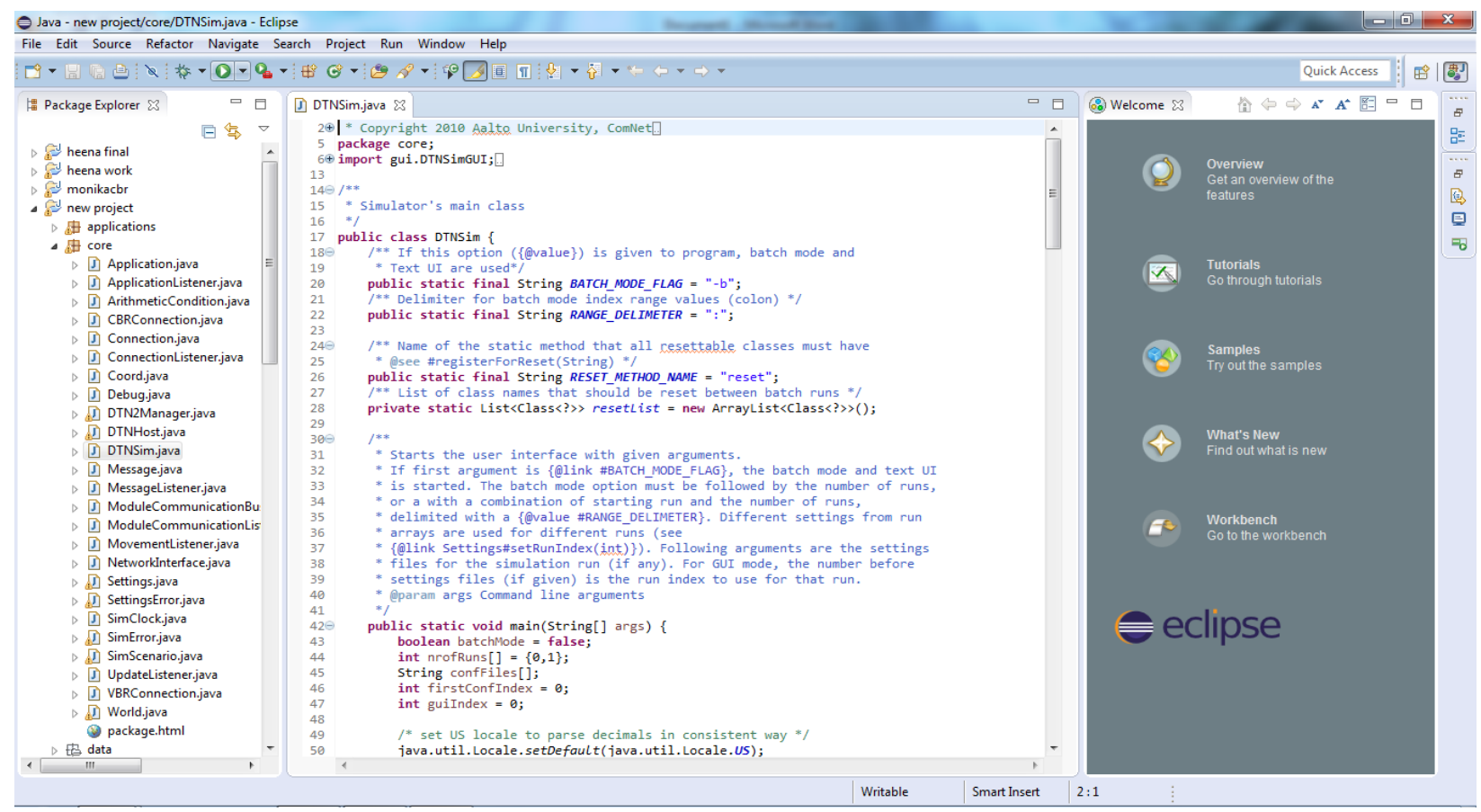

Fig. 11 depicting DTNHOST.JAVA file

To open Simulator open core>DTNsim.java file and click on run button to start simulator.

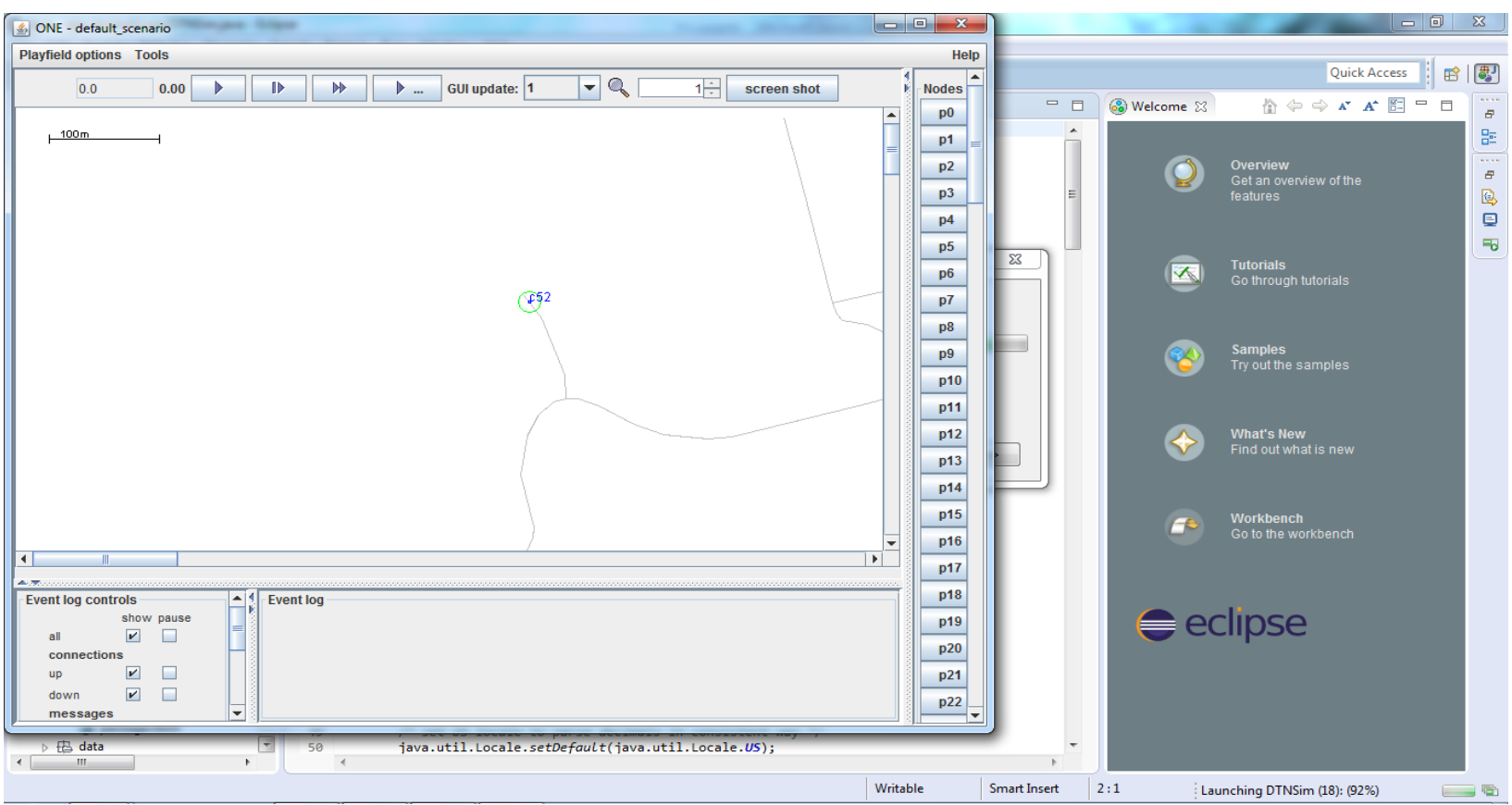

Fig. 12 depicting ONE Simulator GUI

Now you successfully configured and run ONE Simulator. 


\section{RESULTS AND EVALUATION}

ONE simulator provides various existing routing protocols for users to improve these routing protocols or propose a new routing based on existing routing protocols. Here in this section we simulate epidemic routing protocol using ONE simulator. An epidemic routing is an routing in which multiple duplicate messages are transmitted from one hop to other hop at a time. To evaluate results of epidemic routing two performance metrics are used that are throughput and average number of hop count. Throughput determines amount of messages are delivered as Compare to numbers of messages are created. Whereas average hop count determines average of intermediate nodes are presented to transfer a single message.

Table I Throughput V/S Number of Nodes

\begin{tabular}{|r|r|}
\hline \multicolumn{1}{|l|}{$\begin{array}{l}\text { Number } \\
\text { of nodes }\end{array}$} & \multicolumn{1}{l|}{ Epidemic } \\
\hline 20 & 0.2347 \\
\hline 40 & 0.2524 \\
\hline 60 & 0.2652 \\
\hline 80 & 0.2514 \\
\hline 100 & 0.2642 \\
\hline
\end{tabular}

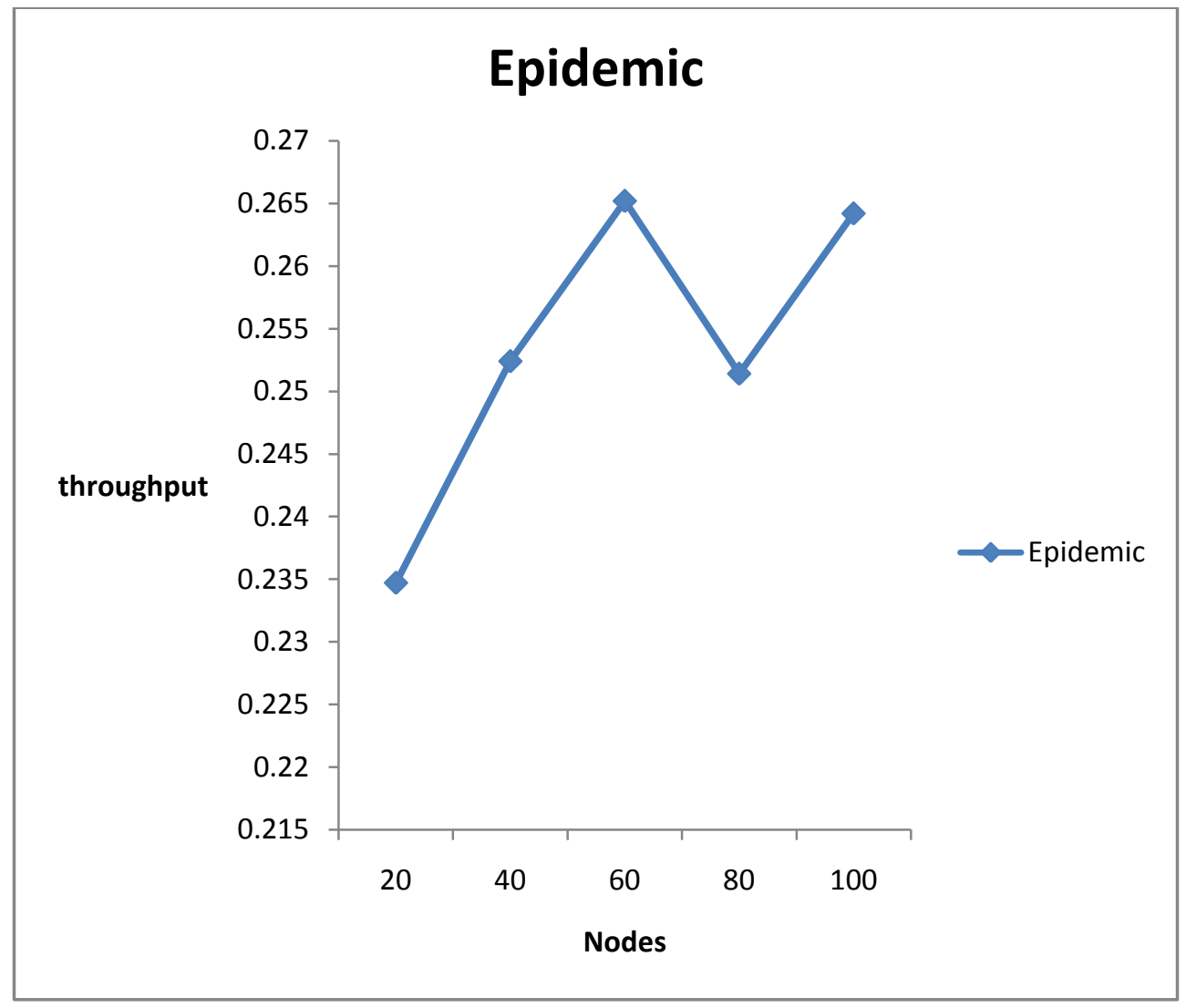

Fig. 13: Throughput v/s number of nodes 
Table 2 Average hop count v/s number of nodes

\begin{tabular}{|r|r|}
\hline \multicolumn{1}{|c|}{$\begin{array}{c}\text { Number of } \\
\text { nodes }\end{array}$} & \multicolumn{1}{|c|}{ Epidemic } \\
\hline 20 & 2.563 \\
\hline 40 & 3.7461 \\
\hline 60 & 4.628 \\
\hline 80 & 5.074 \\
\hline 100 & 6.216 \\
\hline
\end{tabular}

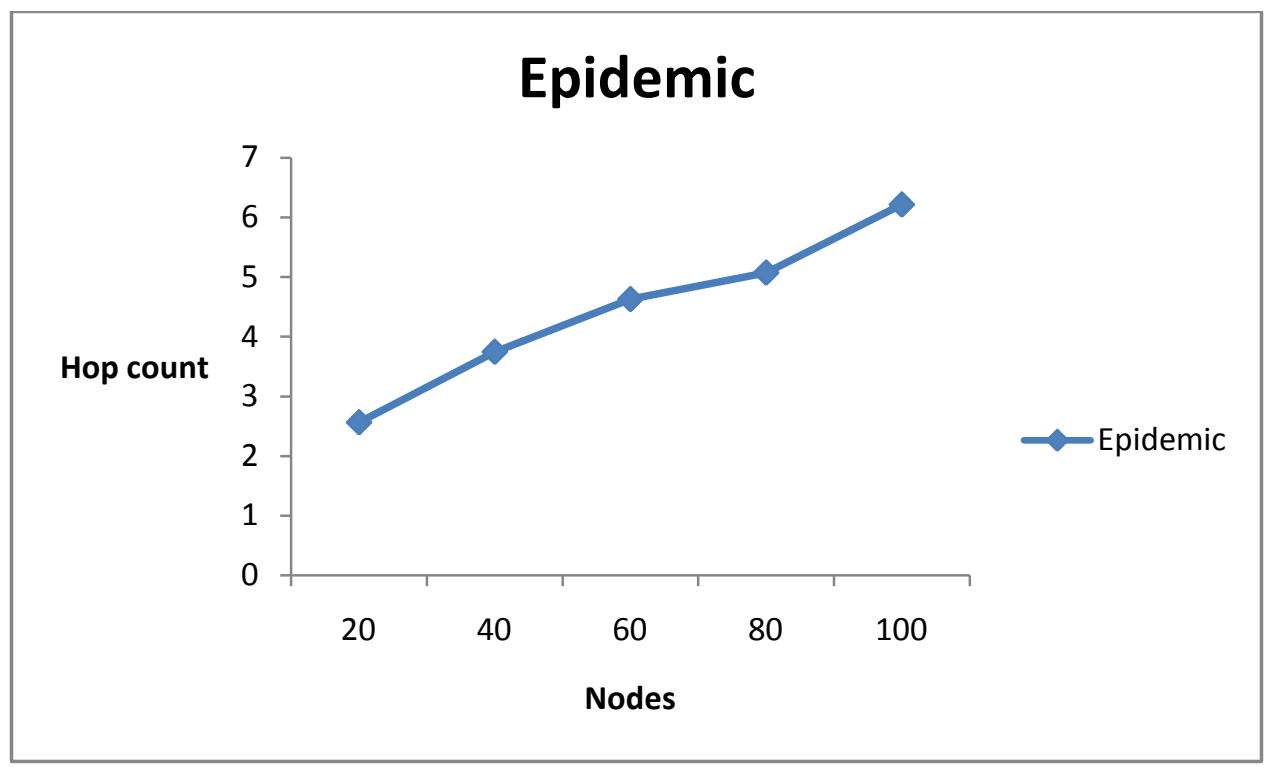

Fig. 14: Average hop count v/s number of nodes

Above table 1 and table 2 shows the resultant output of after simulating epidemic routing protocol. Figure 13 and figure 14 shows that as we increasing nodes during simulation then results varied as nodes increase throughput of network increases similarly intermediate nodes are increase when nodes are increases.

\section{CONCLUSION}

ONE is an excellent simulation tool for wireless networks. It provides simulation environment for DTN also it includes many existing DTN routing algorithms and movement models. In this paper configuration of ONE simulator with eclipse and with proper steps are explained. After that an existing routing protocol named epidemic routing are simulated using simulator. Results are evaluated by varying nodes with performance metrics throughput and average hop count. This helps in programming. In future, I plan to continue working on it and try to review its work flow intensely. After that, I will try to create a technical report that helps to learn ONE simulator. 


\section{REFERENCES}

[1] Barun Saha. The ONE DTN simulator tutorial. Http://delay-tolerant-networks.blogspot.ca/p/one-tutorial.html.

[2] Keränen, A. Opportunistic Network Environment Simulator. Special Assignment report, Helsinki University of Technology, Department of Communications and Networking, May 2008.

[3] Boudec, J.-Y, and Vojnovic, M. Perfect Simulation and Stationarity of a Class of Mobility Models. In Proc. Of IEEE Infocom (2005).

[4] Pelusi, Passarella, and Conti, M. Opportunistic networking: Data forwarding in disconnected mobile ad hoc networks. IEEE Comm. Magazine (Nov 2006).

[5] http://www.netlab.tkk.fi/tutkimus/dtn/theone/

[6] PITKÄNEN, M., AND OTT, J. Enabling Opportunistic Storage for Mobile DTNs. Journal on Pervasive and Mobile Computing 4, 5 (Oct 2008), 579-594.

[7] PITKÄNEN, M. J., KERÄNEN, A., AND OTT, J. Message Fragmentation in Opportunistic DTNs. In Proc. of the $2^{\text {nd }}$ WoWMoM Workshop on Autonomic and Opportunistic Communications (AOC) (2008).

[8] KARVO, J., AND OTT, J. Time scales and delay-tolerant routing protocols. In Proc.c of the ACM MobiCom Workshop on Challenged Networks (CHANTS) (2008).

[9] Ari Keränen, Jörg Ott, Teemu Kärkkäinen, "The ONE Simulator for DTN Protocol Evaluation”, SIMUTools 2009.

[10] Sharma Atul. Opportunistic network environment: A multipurpose simulation tool for wireless networks. Journal of Open Source Developments. 2015; 2(2): 